\title{
Parry-Romberg Syndrome
}

National Institute of Neurological Disorders and Stroke (NINDS)

\section{Source}

National Institute of Neurological Disorders and Stroke (NINDS). Parry-Romberg

Syndrome Information Page.

Parry-Romberg syndrome is a rare disorder characterized by slowly progressive deterioration (atrophy) of the skin and soft tissues of half of the face (hemifacial atrophy), usually the left side. It is more common in females than in males. Initial facial changes usually involve the tissues above the upper jaw (maxilla) or between the nose and the upper corner of the lip (nasolabial fold) and subsequently progress to the angle of the mouth, areas around the eye, the brow, the ear, and the neck. The deterioration may also affect the tongue, the soft and fleshy part of the roof of the mouth, and the gums. The eye and cheek of the affected side may become sunken and facial hair may turn white and fall out (alopecia). In addition, the skin overlying affected areas may become darkly pigmented (hyperpigmentation) with, in some cases, areas of hyperpigmentation and patches of unpigmented skin (vitiligo). Parry-Romberg syndrome is also accompanied by neurological abnormalities including seizures and episodes of severe facial pain (trigeminal neuralgia). The onset of the disease usually begins between the ages of 5 and 15 years. The progression of the atrophy often lasts from 2 to 10 years, and then the process seems to enter a stable phase. Muscles in the face may atrophy and there may be bone loss in the facial bones. Problems with the retina and optic nerve may occur when the disease surrounds the eye. 